1 Title: A comparison of training with a velocity loss threshold 2 or to repetition failure on upper-body strength development in

\title{
Submission Type: Original Investigation
}

\section{Author Information}

\section{Corresponding Author}

Mark J Kilgallon. Sydney Swans Football Club, Sydney,

Australia

Full Mailing Address: Mark Kilgallon, Light Tower 4, SCG, Moore Park, Paddington NSW 2021, Sydney, Australia

Tel: +61 0466540438

E-Mail: markkilgallon@ hotmail.com

Michael J Johnston. British Athletics, University of

Loughborough, UK

Tel: +44 7816670105

E-Mail: mjohnston@britishathletics.org.uk

Liam P Kilduff. Applied Sports Technology Exercise and Medicine Research Centre (A-STEM), Health and Sport

Portfolio, Swansea University, UK

Tel: +44 1792513441

E-Mail: 1.kilduff@swansea.ac.uk

Mark L Watsford. Human Performance Research Centre, Faculty of Health, University of Technology Sydney, Sydney,

Australia

Tel: +61 295145379

E-Mail: Mark.Watsford@uts.edu.au

Abstract Word Count: 250

Article Word Count: 3509

Tables and Figures: 3 x Tables, 1 x Figure

No funding was received for this work 


\section{ABSTRACT}

Purpose: To compare resistance training using a velocity loss threshold with training to repetition failure on upper-body strength parameters in professional Australian footballers.

Methods: 26 professional Australian footballers (23.9 \pm 4.2 years, $189.9 \pm 7.8 \mathrm{~cm}, 88.2 \pm 8.8 \mathrm{~kg})$ tested one-repetition maximum strength (FPmax) and mean barbell velocity at $85 \%$ of 1RM on floor press (FPvel). They were then assigned to two conditions; $20 \%$ velocity loss threshold training (VL; $\mathrm{n}=12$, maximum effort lift velocity) or training to repetition failure (TF; $n=14$, self-selected lift velocity). Subjects trained twice per week for 3 weeks before being reassessed on FPmax and FPvel. Training volume (total repetitions) was recorded for all training sessions. No differences were present between groups on any pre-training measure.

Results: The TF group significantly improved FPmax (105.2 $110.9 \mathrm{~kg},+5.4 \%)$ while the VL group did not $(107.5-109.2 \mathrm{~kg}$, $+1.6 \%)(\mathrm{p}<0.05)$. Both groups significantly increased the FPvel $\left(0.38-0.46 \mathrm{~m} . \mathrm{s}^{-1},+19.1 \%\right.$ and $0.37-0.42 \mathrm{~m} . \mathrm{s}^{-1},+16.7 \%$, respectively) with no between-group difference evident ( $>0.05$ ). The TF group performed significantly more training volume (12.2 vs. 6.8 repetitions per session, $\mathrm{p}<0.05)$.

Conclusion: Training to repetition failure improved FPmax while training using a velocity loss threshold of $20 \%$ did not. Both groups demonstrated similar improvements in FPvel despite the VL group performing $45 \%$ less total training volume than the TF group. The reduction in training volume associated with implementing a $20 \%$ velocity loss threshold may negatively impact the development of upper-body maximum strength while still enhancing submaximal movement velocity.

Key Words: Linear position transducer, velocity based training, preseason, concurrent training, training dose response

\section{INTRODUCTION}

Australian football is a contact sport that involves athletes performing repeated bouts of high intensity activity (e.g. sprinting, jumping, tackling) interspersed with periods of lower intensity movements (e.g. jogging, walking). ${ }^{1}$ While aerobic endurance is a central determinant of performance due to the extreme running demands of the game, ${ }^{2}$ high levels of strength are also required to perform a variety of movements such as bumping, tackling, wrestling and fending off of opponents when contesting possession., ${ }^{2,3}$ Upper-body strength is positively related to team selection in elite junior players, ${ }^{4}$ while strong associations have been reported between 1 repetition maximum (1RM) bench press and a number of in game statistics such as contested possessions, hard ball gets, physical pressure acts and clearances for certain positions in elite senior players. ${ }^{5}$ From a 
performance enhancement perspective, the development of upper-body strength and power qualities in AFL footballers would appear intuitive.

Traditionally effective strength program design involves the manipulation of training variables such as training intensity, volume, rest periods, and exercise selection. ${ }^{6}$ However in recent years a number of velocity based training (VBT) methods have evolved whereby velocity has become an important variable in the programming process. ${ }^{7}$ Based on the observation that barbell velocity loss across repetitions occurs in a predictable, linear pattern when concentric actions are performed with maximal intent, ${ }^{6,8}$ coaches can now accurately quantify the acute level of fatigue during a set in real-time and utilise this metric to regulate training stress. One such method involves the use of velocity loss thresholds whereby an athlete will terminate a set once a predetermined level of barbell velocity loss has occurred. ${ }^{9}$ This approach facilitates athletes training at higher average movement velocities and may better stimulate rapid force production adaptations ${ }^{9}$ as it has been demonstrated that actual movement velocity of training influences subsequent neuromuscular responses. ${ }^{10}$ This method also potentially reduces the risk of overtraining by reducing the acute metabolic stress, hormonal response, muscle damage and overall fatigue induced by traditional methods like training to repetition failure. ${ }^{11,12}$

To-date the use of low velocity loss thresholds (15-20\% range) have been reported to be equally, or more effective in comparison to high velocity loss thresholds (30-40\% range) at optimising training volume, movement speeds and subsequent strength and power gains on the lower-body measures in resistance trained males ${ }^{9}$ and male professional soccer players. ${ }^{12}$ Significant gains in upper-body strength have been reported in resistance trained males employing a $20 \%$ velocity loss threshold after 3 weeks of training, but not in those training to repetition failure. ${ }^{13}$ To date no studies have investigated the effect of velocity loss thresholds on upper-body strength in athletic populations. Given the often extreme physical demands involved in preparing for professional sports, investigating the efficacy of training methods that could potentially induce positive adaptations in strength and movement velocity while reducing unnecessary training stress is warranted in this cohort.

Therefore, the purpose of the current study was to investigate the effects of three weeks of resistance training with a $20 \%$ velocity loss threshold vs. repetition failure on upper-body strength in professional Australian footballers. It was hypothesised that training with a velocity loss threshold would lead to similar or 
superior gains in maximum strength and submaximal movement velocity than training to repetition failure.

\section{METHODS}

\section{Subjects}

A total of 28 professional footballers from one senior football team playing in the Australian Football League (AFL) participated in the study which was conducted during their regular preseason training program (See Table 1). Two subjects were forced to drop out due to injury incurred during on-field sessions. Therefore, 26 subjects completed the study (mean \pm SD; age: $23.9 \pm 4.2$ years; height: $189.9 \pm 7.8 \mathrm{~cm}$; body mass: $88.2 \pm 8.8 \mathrm{~kg}$; senior games played: $70.5 \pm 18$ ). The inclusion criteria required that all subjects were healthy and had been engaged in continuous resistance training for a minimum of one year prior to the study start date. Based on previous research assessing velocity loss thresholds in resistance-trained individuals, ${ }^{13}$ a large effect size was anticipated for the betweengroup differences for the primary variable (upper-body strength). Therefore, with a power level of $1-\beta=0.80$, the minimum sample size was deemed to be 12 participants per group. ${ }^{14}$ Approval for the research was granted by the Human Research Ethics Committee of the

\section{Design}

This study employed a non-randomised, parallel group, pre-post experimental design to compare the effects of 3 weeks of training with a $20 \%$ velocity loss threshold or training to repetition failure on measures of upper-body strength. All subjects were tested for upper-body strength (1RM floor press [FPmax]) and submaximal lift velocity (maximum effort velocity test at $85 \%$ of their established 1RM [FPvel]). They were then assigned to one of two training groups (velocity loss threshold or training to repetition failure) where they performed two sessions of upper-body pressing per week for 3-weeks. Five days after the completion of the training all participants were retested and the results were analysed for any differences in strength, movement velocity and training volume (repetition count) between the groups. All testing and training was performed as part of scheduled preseason strength training sessions (see Table 1). Post-testing occurred at a Day 1 session to ensure all subjects had 2 days of rest immediately prior. All subjects received an identical protein supplement immediately after all strength training sessions. No other nutritional supplement strategies were employed for the duration of the study. 


\section{Procedures}

\section{Football Training \& Conditioning}

Total training time and total running distance were recorded using GNSS units sampling at $10 \mathrm{~Hz}$ ('Optimeye S5', Catapult Sports, Melbourne, Australia). Intra-class correlation coefficients (ICCs) for Catapult GNSS devices have demonstrated high to very high reliability ( $r=0.86-0.99)$ for distances covered at low-, high-, and very high-speed running intensities. $^{15}$

\section{Anthropometry}

Body mass was recorded using a calibrated portable digital scale (Tanita, Wedderburn, Japan) to the nearest $0.01 \mathrm{~kg}$, with players advised to remove footwear and wear light fitting clothing. Height was measured from the floor to the top of the skull using a portable stadiometer (Ecomed, Seca, Australia) and measured to the nearest $0.1 \mathrm{~cm}$.

\section{Upper-body strength}

The floor press exercise was selected for familiarisation purposes as this was the primary measure of upper-body strength used by the team and all subjects were well trained in the movement (floor press training experience: $3.3 \pm 1.3$ years). Floor press 1RM (FPmax) testing was performed following a standardised warm up. The subjects performed an initial set of 5 repetitions at $60 \%$ of their estimated 1RM (based upon recent training history and previous maximum test results). Load was increased to $75 \%$ for 3 repetitions, $85 \%$ for 2 repetitions and $95 \%$ for 1 repetition. At this stage the researcher dictated incremental load increases until 1RM was achieved with correct technique allowing 4-5 minutes of rest between each attempt. The exercise was performed with legs straight and no hip lift was permitted. Subjects were instructed to lower the barbell with control until their elbows touched the floor, pause in the bottom position for a 2 second count verbally controlled by the lift spotter, and then press the barbell to full lockout without assistance. The FPmax procedure displayed excellent levels of test-retest reliability when 13 players were assessed twice over a two week period $\left(\mathrm{ICC}_{2,1}=0.99\right.$ [95\% CI 0.98$1.00])$.

\section{Velocity}

Mean barbell velocity was measured using a linear position transducer (GymAware PowerTool; Kinetic Performance Technology, Canberra, Australia) attached to the loading sleeve of the barbell. This system has previously been reported to provide valid measures of mean concentric barbell velocity ${ }^{16}$ while the specific FPvel testing procedure used in the current study revealed an excellent level of test-retest reliability when 
11 players were assessed twice over a two week period ( $\mathrm{ICC}_{2,1}$

$251=0.91$ [95\% CI 0.65-0.98)). Following the FPmax test subjects

252 were given a 5 minute rest before establishing their 85\%1RM

253 floor press mean velocity (FPvel) by performing one set of 2 repetitions at $85 \%$ of their $1 \mathrm{RM}$. Subjects were instructed to pause for 2 seconds in the bottom position before vertically pressing the barbell concentrically as fast and explosively as possible across the full range of motion to full lockout.

258 Performing a 2 second pause prior to the concentric portion of a

259 lift has previously been shown to improve reliability during

260 isoinertial strength testing. ${ }^{17}$ Strong verbal encouragement and

261 velocity feedback was provided as this has been shown to

262 improve athlete motivation and performance in strength tasks

263 that involve measuring movement velocity. ${ }^{18}$ The fastest of the

264 two repetitions was used for further analysis. To enable direct

265 comparison, velocity testing was performed at the same

266 absolute load following the training period (85\% of pre-test

267 FPmax). There was one minor difference in testing order at the

268 post-test. Since the test load was already established from pre-

269 testing, the post-test FPvel was performed during the warm up

270 progression and not following the establishment of the 1RM.

271 To ensure fatigue had not negatively impacted the pre-testing

272 FPvel, individual pre-test velocity was reset at the first training

273 session if a subject exceeded their pre-testing score.

All upper-body strength and velocity testing was performed under the direct supervision of the lead investigator, at the same venue and at the same time of day for each subject ( \pm 2 hours).

\section{Training Interventions}

The descriptive characteristics of the resistance training programs are presented in Table 2. Gym sessions were performed in the afternoon and were always preceded by fieldbased skills and endurance training followed by a 3-hour recovery period. All strength sessions were supervised by the lead investigator (UKSCA accredited coach). Floor press was performed twice per week for the 3-week duration of the study similar to previous velocity loss threshold protocol design. ${ }^{13}$ All floor press repetitions were paused for two seconds in the bottom position as per the test protocol. Rest periods of 3-4 minutes were prescribed between sets. No other pressing movements were performed for the duration of the project. Lift volumes and relative lift intensities were identical for both groups on all other strength exercises performed as part of the preseason strength program.

A non-randomised procedure was used for allocating the training groups due to the logistical demands associated with a professional sports team. Groups were selected based on program scheduling priorities (positional/tactical meetings etc), 
however all specific football training and conditioning programs were controlled for load and homogenous between the groups for the duration of the study.

Train to repetition failure (TF) Group: Failure was defined as the subject being unable to perform another repetition without assistance. No velocity monitoring was performed and subjects were instructed to perform the concentric phase of movement at their normal, self-selected speed. The total number of repetitions performed on all worksets at $85 \% 1 \mathrm{RM}$ were recorded.

Velocity loss (VL) Group: All sets were performed with real time velocity feedback provided using linear position transducers (GymAware PowerTool; Kinetic Performance Technology, Canberra, Australia). Individual velocity loss thresholds for training were set at $20 \%$ below the fastest repetition from their pre-test FPvel. ${ }^{13}$ Once a repetition was performed below this threshold velocity the participant was alerted via an auditory tone and the set was immediately terminated. The total number of repetitions performed on all worksets at $85 \% 1 \mathrm{RM}$ were recorded with the final repetition of each set where the participant failed to achieve the required velocity being included in this value.

\section{***Insert Table 2 about here ${ }^{* * *}$}

\section{Statistical Analysis}

For all variables, values are presented as mean \pm standard deviation (SD). The standard error of the measurement was also calculated using the formula $\mathrm{SEM}=\mathrm{SD} /(1-\mathrm{ICC})^{-2}$. T-tests were completed to examine baseline intergroup differences (independent samples test), and pre to post intragroup football training and conditioning volumes (paired tests). Data was analysed using a $2 \times 2$ (Time x Group) factorial ANOVA. Where a significant interaction effect was present, paired sample t-tests were completed to examine pre- to post-training intragroup differences. Differences in repetition count from the first to the final week of training were examined using paired sample t-tests for the intragroup analyses and independent sample t-tests for the intergroup comparisons. The minimum effective dose for the training was examined by correlating the average session training volume and the percentage change in strength elicited from the training program. Linear regression analysis yielded equations for the slopes of the trendlines of each condition to enable comparisons between training methods. Effect sizes (ES) were calculated using partial eta squared $\left(\eta^{2}\right)$ for the factorial ANOVA with magnitudes defined as small $(<0.06)$, moderate (0.06-0.14) and large (>0.14). Cohen's $d$ was calculated to quantify the ES for intragroup differences and classified as small $(<0.50)$, moderate $(0.50-0.79)$ and large $(>0.80) .{ }^{19}$ Data analysis 
was completed using SPSS 25.0 (Chicago, IL, USA) and statistical significance for all tests was set at $p<0.05$.

\section{RESULTS}

\section{Baseline}

There were no significant differences between the TF and VL groups reported before training for any variables.

\section{***Insert Table 3 about here ${ }^{* * *}$}

Football Training \& Conditioning

No differences were recorded between groups for total training time $(\mathrm{p}=0.50)$ or total running distance $(\mathrm{p}=0.50)$ over the course of the study.

\section{$\underline{\text { Body Mass }}$}

Body mass remained unchanged from pre to post-testing in the TF and VL groups (Table 3).

\section{Strength}

A significant main effect for time was reported for FPmax $\left(p<0.01, \eta^{2}=0.43\right)$. A significant group $x$ time interaction effect was evident between training groups $\left(p=0.03, \eta^{2}=0.19\right)$ with training resulting in significant increases in maximum strength for the TF group $(\mathrm{p}<0.01)$ but not for the VL group $(\mathrm{p}=0.07)$ (Table 3). A moderate ES was reported for the improvement in strength in the TF group and a small ES for the changes in the VL group (Table 3).

\section{Velocity}

There was no group $\mathrm{x}$ time interaction effect evident between training groups $\left(p=0.63, \eta^{2}=0.01\right)$. A significant main effect for time was reported for FPvel $\left(p<0.01, \eta^{2}=0.52\right)$ with training resulting in significant increases in FPvel for the TF group $(\mathrm{p}<0.01)$ and the VL group $(\mathrm{p}<0.01)$ (Table 3). A large ES was reported for both groups for this variable (Table 3 ).

\section{Training Volume}

The TF group $(p<0.01)$ and VL group $(p=0.03)$ both significantly increased the total number of repetitions performed per set from week 1 to week 3 (Table 3 ). The total repetitions completed per training session was also different between each group for week $1(\mathrm{p}<0.01)$ and week $3(\mathrm{p}<0.01)$ with the TF group recording higher values. As demonstrated in Figure 1, there was a tendency for a greater change in strength with a greater number of repetitions completed during training. Based on the y-intercept and positive gradient of each plot, an effective minimum training dose appears to exist for both conditions. 
***Insert Figure 1 about here***

\section{DISCUSSION}

The main finding of this study was that short-term resistance training using a $20 \%$ velocity loss threshold did not lead to a significant increase in FPmax while training to repetition failure did in professional Australian footballers. Interestingly, both methods of training increased FPvel, despite the velocity loss threshold group performing $45 \%$ less total training volume. To our knowledge this is the first time the efficacy of using a velocity loss threshold protocol has been investigated on measures of upper-body strength in professional team sports athletes.

Contrary to our hypothesis, resistance training utilising a $20 \%$ velocity loss threshold did not increase FPmax $(+1.6 \%)$ while training to repetition failure led to an improvement $(+5.4 \%)$ (Table 3). Training to failure may allow recruitment and overload of a larger pool of active motor units potentially leading to greater strength development ${ }^{20}$ while the increased metabolic stress, hormone response, and muscle damage involved may mediate hypertrophic adaptations. ${ }^{21}$ While no changes in body mass occurred, a more detailed anthropometric assessment would be required to ascertain whether the FPmax increase occurred independently of muscle hypertrophy. The magnitude of strength gains in the TF group is consistent with the findings of Drinkwater et al. ${ }^{20}$ who reported a $9.6 \%$ increase in bench press $1 \mathrm{RM}$ after 6 weeks of training to failure in elite junior basketball and soccer players. This rate of improvement is similar to the current study when the longer time frame involved is taken into account.

The absence of strength changes for the VL group are in contrast to those of Padulo et al. ${ }^{13}$ who reported a $10.2 \%$ increase in $1 \mathrm{RM}$ bench press using a $20 \%$ velocity loss threshold in resistance trained males. Training mode, lift intensity, velocity loss threshold, frequency and program duration were all similar to the current study. However total training volume did differ, with the current study utilising a lower number of work sets per session (two fixed work sets) as opposed to continuous sets until subjects were unable to complete a single effective repetition under either condition. ${ }^{13}$ This difference may contribute to the contradictory findings between the studies and potentially highlight that an inverted U-shaped relationship may exist between training volume and adaptations. ${ }^{12}$ While two work sets may be sufficient to stimulate strength gains after 3 weeks of training when the sets are performed to failure, it may not provide an adequate training volume to stimulate strength gains when utilising a $20 \%$ velocity loss threshold, and concurrently undertaking endurance training. The existence of population specific dose-response 
relationships with respect to training volume and strength development in untrained, recreationally trained, and trained athletic cohorts have been identified. ${ }^{22}$ Given the focus on movement velocity, the two workset protocol utilised by the VL group in the current study did not meet the mean training volume required to maximise strength gains for a trained athletic population. ${ }^{22}$ Examination of the individual training volume data in conjunction with the information pertaining to strength changes (Figure 1) also highlights that a minimum effective training volume for each training condition may exist. Future research should aim to scrutinise this aspect of velocity based training more closely.

The VL and TF protocols both resulted in a significant increase in FPvel $(+16.7 \%$ and $+19.1 \%$ respectively), with large effect sizes for both conditions ( $d=1.33$ and 1.11 , respectively). The increase in FPvel for the TF group may be partially explained by the increase in FPmax, resulting in a reduction in the relative intensity that the pre-test $85 \% 1 \mathrm{RM}$ load represented. However, the improvement in FPvel in the VL group occurred independent of a change in maximum strength. Furthermore, this increase in submaximal lifting velocity occurred despite the VL group performing $45 \%$ less training volume per session than the TF group. This finding is similar to previous research assessing velocity adaptations to velocity loss threshold training in untrained males. ${ }^{9}$ Specifically, it has been reported that training groups using $20 \%$ or $40 \%$ velocity loss thresholds across a range of loads for 8 weeks on back squats both significantly increased mean propulsive velocity at heavy loads $(+12.7 \%$ vs $+13.7 \%)$ despite the $20 \%$ velocity loss group actually performing $40 \%$ less total training volume. ${ }^{9}$

While the professional status of the athletes involved in the current study gives unique insight into the adaptation of elite level field sport athletes to velocity based training methods, being able to perform such research comes with a number of practical limitations due to the logistical and performance demands of a professional football program. For instance, as all subjects were involved in a preseason training phase, they were required to concurrently train strength and endurance qualities which could have compromised the strength and/or velocity adaptations of both groups ${ }^{23}$. Due to scheduling constraints of the football program it was also not possible to randomise the training group allocation, although no difference was reported between groups on any outcome measures at pre-testing. Finally, it can be argued that the relatively short program duration (three weeks) may not have provided sufficient training time for increases in maximum strength to manifest using a $20 \%$ velocity loss threshold. However significant 1RM strength gains in bench press have been reported previously over a similar time frame ${ }^{13}$ 
500

501

502

503

504

505

506

507

508

509

510

511

512

513

514

515

516

517

518

519

520

521

522

523

524

525

526

527

528

529

530

531

532

533

534

535

536

537

538

539

540

541

542

543

544

545

546

547

548

and both groups did significantly improve FPvel in the current study. It is also worth noting that compliance was $100 \%$ for all subjects ensuring training exposure was maximised despite the constraints of the study design.

\section{PRACTICAL APPLICATIONS}

This study suggests resistance training to repetition failure can be an effective method to optimise upper-body maximum strength during a short, intensive preseason training block. In contrast, the evident velocity adaptations imply that strength and conditioning coaches can continue to stimulate strength-speed adaptations with significantly reduced training volumes when a $20 \%$ velocity loss threshold is utilised. This may be particularly relevant during short peaking phases or during intensive competition blocks when athletes have less available time to train in the weight-room and practitioners attempt to minimise excessive fatigue. Finally, while the use of a $20 \%$ velocity loss threshold did not improve maximum strength, further research into velocity loss threshold strategies is warranted to better understand specific dose-response dynamics.

\section{CONCLUSIONS}

A three-week training program incorporating a velocity loss threshold did not increase upper-body FPmax in professional footballers while training to repetition failure led to an improvement. Further, while both training modalities enhanced FPvel, the velocity loss threshold group achieved this adaptation despite performing significantly less total training volume than the training to repetition failure group. These findings have implications for strength and conditioning practitioners looking to implement velocity loss threshold training methods with athletic populations.

\section{ACKNOWLEDGMENTS}

The Football Club supported this research. No funding was received for this project. The authors have no conflicts of interest to declare.

\section{REFERENCES}

1. Johnston RD, Black GM, Harrison PW, Murray NB, and Austin DJ. Applied sport science of Australian football: A systematic review. Sports Med 2018;48: 1673-1694.

2. Gray AJ, and Jenkins DG. Match analysis and the physiological demands of Australian football. Sports Med. 2010;40: 347-360.

3. Hrysomallis C, and Buttifant D. Influence of training years on upper-body strength and power changes during 


\begin{tabular}{|c|c|c|}
\hline 549 & & mpetitive season for professional Australian ru \\
\hline 550 & & football players. J Sci Med Sport. 2012;15: 374-378. \\
\hline 551 & 4. & Keogh J. The use of physical fitness scores and \\
\hline 52 & & anthropometric data to predict selection in an elite \\
\hline 53 & & under 18 Australian rules football team. J Sci Med \\
\hline 24 & & Sport. 1999;2: 125-133. \\
\hline 55 & 5. & Stares J, Dawson B, Heasman J, and Rogalski B. \\
\hline 56 & & Relationship between pre-season strength and power \\
\hline 57 & & measures and performance in elite Australian Football. \\
\hline 88 & & Int J Perf Anal Spor. 2015;15: 777-793. \\
\hline & 6. & González-Badillo JJ, and Sánchez-Medina L. \\
\hline 50 & & Movement velocity as a measure of loading intensity in \\
\hline 51 & & resistance training. Int J Sports Med. 2010;31: 347-352. \\
\hline 52 & 7. & Weakley J, Mann B, Banyard H, McLaren S, Scott T, \\
\hline & & Garcia-Ramos A. Velocity-Based Training. From \\
\hline 54 & & Theory to Application. Strength Cond J. 2020; Publish \\
\hline 65 & & Ahead of Print. \\
\hline 66 & 8. & Izquierdo M, González-Badillo J, Häkkinen K, et al. \\
\hline 57 & & Effect of loading on unintentional lifting velocity \\
\hline 58 & & declines during single sets of repetitions to failure \\
\hline 59 & & during upper and lower extremity muscle actions. Int $J$ \\
\hline 70 & & Sports Med. 2006;27: 718-724. \\
\hline 71 & 9. & Pareja-Blanco F, Rodríguez-Rosell D, Sánchez-Medina \\
\hline 72 & & $\mathrm{~L}$, et al. Effects of velocity loss during resistance \\
\hline 73 & & training on athletic performance, strength gains and \\
\hline 74 & & muscle adaptations. Scand J Med Sci Spor. 2017;27: \\
\hline 75 & & $724-735$ \\
\hline 76 & 10. & Kaneko M. Training effect of different loads on the \\
\hline 77 & & force-velocity relationship and mechanical power \\
\hline 78 & & output in human muscle. Scand J Sports Sci. 1983;5: \\
\hline 79 & & $50-55$ \\
\hline 580 & 11. & Izquierdo-Gabarren M, Exposito RGDT, Garcia- \\
\hline 581 & & Pallares J, Sanchez-Medina L, De Villarreal ES, and \\
\hline 82 & & Izquierdo M. Concurrent endurance and strength \\
\hline 583 & & training not to failure optimizes performance gains. \\
\hline 584 & & Med Sci Sports Exerc. 2010;42: 1191-1199. \\
\hline 585 & 12. & Pareja-Blanco F, Sánchez-Medina L, Suárez-Arrones L, \\
\hline 0 & & and González-Badillo JJ. Effects of velocity loss during \\
\hline 87 & & resistance training on performance in professional \\
\hline 588 & & soccer players. Int J Sports Physiol Perform. 2017;12: \\
\hline 58 & & $512-519$ \\
\hline 590 & 13. & Padulo J, Mignogna P, Mignardi S, Tonni F, and \\
\hline$\pi$ & & D'ottavio S. Effect of different pushing speeds on bench \\
\hline 59 & & press. Int J Sports Med. 2021;33: 376-380. \\
\hline 593 & 14. & Kraemer HC, and Thiemann S. How many subjects?: \\
\hline 594 & & Statistical power analysis in research. Newbury Park, \\
\hline 59 & & CA: Sage; 1987. \\
\hline - & 15. & Johnston RJ, Watsford ML, Kelly SJ, Pine MJ, Spurrs \\
\hline & & RW. Validity and interunit reliability of $10 \mathrm{~Hz}$ and 15 \\
\hline
\end{tabular}


602

603

604

605

606

607

608

609

610

611

612

613

614

615

616

617

618

619

620

621

622

623

624

625

626

627

628

629

630

631

632

633

$\mathrm{Hz}$ GPS units for assessing athlete movement demands. J Strength Cond Res. 2014;28(6): 1649-1655.

16. Dorrell HF, Moore JM, Smith MF, and Gee TI. Validity and reliability of a linear positional transducer across commonly practised resistance training exercises. $J$ Sports Sci. 2019;37: 67-73.

17. Pallarés JG, Sánchez-Medina L, Pérez CE, De La CruzSánchez E, and Mora-Rodriguez R. Imposing a pause between the eccentric and concentric phases increases the reliability of isoinertial strength assessments. $J$ Sports Sci. 2014;32: 1165-1175.

18. Weakley J, Wilson K, Till K, Banyard H, Dyson J, Phibbs P, Read D, and Jones B. Show Me, Tell Me, Encourage Me: The Effect of Different Forms of Feedback on Resistance Training Performance. $J$ Strength Cond Res. 2018;00(00): 1-7.

19. Cohen J. Statistical Power Analysis for the Behavioral Sciences. 2nd edn. Hillsdale, NJ: Lawrence Erlbaum Associates; 1988.

20. Drinkwater EJ, Lawton TW, Lindsell RP, Pyne DB, Hunt PH, and Mckenna MJ. Training leading to repetition failure enhances bench press strength gains in elite junior athletes. J Strength Cond Res. 2005;19: 382388.

21. Schoenfeld BJ. The mechanisms of muscle hypertrophy and their application to resistance training. J Strength Cond Res. 2010;24: 2857-2872.

22. Peterson MD, Rhea MR, and Alvar BA. Application of the Dose-Response for Muscular Strength Development: A Review of Meta-Analytic Efficacy and Reliability for Designing Training Prescription. $J$ Strength Cond Res. 2005;19: 950-958.

23. Fyfe JJ, Bishop DJ, and Stepto NK. Interference between concurrent resistance and endurance exercise: molecular bases and the role of individual training variables. Sports Med. 2014;44: 743-762. 